\title{
GhWRKY15, a member of the WRKY transcription factor family identified from cotton (Gossypium hirsutum L.), is involved in disease resistance and plant development
}

Feifei Yu', Yifeng Huaxia', Wenjing $\mathrm{Lu}^{1}$, Changai Wu${ }^{1}$, Xuecheng $\mathrm{CaO}^{2^{*}}$ and Xingqi Guo ${ }^{{ }^{* *}}$

\begin{abstract}
Background: As a large family of regulatory proteins, WRKY transcription factors play essential roles in the processes of adaptation to diverse environmental stresses and plant growth and development. Although several studies have investigated the role of WRKY transcription factors during these processes, the mechanisms underlying the function of WRKY members need to be further explored, and research focusing on the WRKY family in cotton crops is extremely limited.
\end{abstract}

Results: In the present study, a gene encoding a putative WRKY family member, GhWRKY15, was isolated from cotton. GhWRKY15 is present as a single copy gene, and a transient expression analysis indicated that GhWRKY15 was localised to the nucleus. Additionally, a group of cis-acting elements associated with the response to environmental stress and plant growth and development were detected in the promoter. Consistently, northern blot analysis showed that GhWRKY15 expression was significantly induced in cotton seedlings following fungal infection or treatment with salicylic acid, methyl jasmonate or methyl viologen. Furthermore, GhWRKY15overexpressing tobacco exhibited more resistance to viral and fungal infections compared with wild-type tobacco. The GhWRKY15-overexpressing tobacco also exhibited increased RNA expression of several pathogen-related genes, NONEXPRESSOR OF PR1, and two genes that encode enzymes involved in ET biosynthesis. Importantly, increased activity of the antioxidant enzymes POD and APX during infection and enhanced expression of NtAPXI and NtGPX in transgenic tobacco following methyl viologen treatment were observed. Moreover, GhWRKY15 transcription was greater in the roots and stems compared with the expression in the cotyledon of cotton, and the stems of transgenic plants displayed faster elongation at the earlier shooting stages compared with wide type tobacco. Additionally, exposure to abiotic stresses, including cold, wounding and drought, resulted in the accumulation of GhWRKY15 transcripts.

Conclusion: Overall, our data suggest that overexpression of GhWRKY15 may contribute to the alteration of defence resistance to both viral and fungal infections, probably through regulating the ROS system via multiple signalling pathways in tobacco. It is intriguing that GhWRKY15 overexpression in tobacco affects plant growth and development, especially stem elongation. This finding suggests that the role of the WRKY proteins in disease resistance may be closely related to their function in regulating plant growth and development.

Keywords: GhWRKY15, Cotton, Disease resistance, SA, ROS, Plant development

\footnotetext{
* Correspondence: xccao@sdau.edu.cn; xqguo@sdau.edu.cn

${ }^{1}$ State Key Laboratory of Crop Biology, College of Life Sciences, Shandong Agricultural University, Taian, Shandong 271018, P. R. China

${ }^{2}$ College of Information Science and Engineering, Shandong Agricultural

University, Taian, Shandong 271018, P. R. China
} 


\section{Background}

Although sessile plants lack a circulating, somatically adaptive immune system, they have developed specific mechanisms to respond to diverse environmental signals that may cause stress and restrict growth and development. Among these mechanisms, many genes that either directly protect plants against stress or further control the expression of other target genes are induced or repressed [1]. Therefore, plant defence responses are regulated by a network of signalling pathways that finetune the transcriptional activation of defence-related genes. Among these complex responses, it has been demonstrated that the transcriptional activation of defence-related genes requires the expression of particular transcription factors and/or alterations in their DNAbinding activity $[2,3]$.

The WRKY family is one of the largest families of transcription factors in plants. Since the first member was isolated in sweet potato, an increasing number of WRKY members have been identified, including 74 members in Arabidopsis thaliana and 109 members in Oryza sativa $[4,5]$. The defining characteristic of WRKY transcription factors is their DNA-binding domain, known as the WRKY domain, which is composed of a highly conserved peptide sequence (WRKYGQK) and a zinc-finger motif, (either $\mathrm{Cx}_{4-5} \mathrm{Cx}_{22-23} \mathrm{HxH}$ or $\mathrm{Cx}_{7} \mathrm{Cx}_{23} \mathrm{HxC}$ ) [6]. Moreover, the conservation of the WRKY domain is mirrored by the conservation of cognate cis-acting Wbox elements $((\mathrm{T}) \mathrm{TGAC}(\mathrm{C} / \mathrm{T}))$ in the target promoter region, which are essential for the function and binding of the WRKY protein [4]. The number of highly conserved WRKY domains and the structure of the zincfinger motif have resulted in the division of the WRKY superfamily into three distinct groups (I, II and III), and group II can be further split into five distinct subgroups (IIa to IIe) based on the presence of additional short conserved structural motifs [4].

Previous studies have examined the roles of plant WRKY proteins in response to pathogens, and WRKY proteins can function as either positive or negative regulators of the defence response. Constitutive overexpression of WRKY7O results in increased resistance to pathogens and the constitutive expression of salicylic acid (SA)-induced pathogen-related (PR) genes. Generally, the expression of AtWRKY70 is activated by SA and repressed by JA [7]. Furthermore, the enhanced resistance of CaWRKY1-silenced chilli pepper leaves to Xanthomonas axonopodis pv. vesicatoria suggests that CaWRKY1 acts as a negative regulator of the defence response [8]. Interestingly, a dual function in defence signalling has been suggested for AtWRKY53 and AtWRKY41 [9,10]. WRKY41-overexpressing Arabidopsis exhibited enhanced resistance to Pseudomonas syringae but increased susceptibility to Erwinia carotovora [10].
Mechanistically, to increase the defence response, WRKY transcription factors frequently upregulate the expression of several pathogen-related genes and NONEXPRESSOR OF PR1 (NPR1) by binding specifically to the W-box element in their promoter region $[3,11]$. The expression of PR proteins, including antimicrobial chitinases and glucanases that directly destroy the cell walls of fungal pathogens, is temporarily and spatially activated following pathogen recognition [12]. NPR1 is a key regulator of the SA-dependent defence response and systemic acquired resistance (SAR), and plants underexpressing NPR gene are more susceptible to infection with pathogens [3]. These data suggest that the mechanism underlying the regulation of the defence response by WRKY transcription factors is fairly complex and needs to be examined further.

Importantly, GmWRKY27 proteins in soybean (Glycine $\max$ L.) probably form homodimers and can efficiently activate reporter gene expression in a two-hybrid assay [13]. Interactions among WRKY18, WRKY40 and WRKY60 can result in both homocomplexes and heterocomplexes, thereby allowing for adaptation to different microbial pathogens [14]. Such protein-protein interactions may affect properties that are important for the regulatory functions of these proteins [14].

Few studies have examined the role of the WRKY family of transcription factors in plant development. The overexpression of GmWRKY13 promotes lateral root development, and this promotion is probably the result of the activation of the downstream gene ARF6, a component of the auxin signalling pathway [13]. Some WRKY transcription factors also function in seed development, dormancy and germination; leaf senescence; and trichome development, which are mostly dependent upon the abscisic acid (ABA) and gibberellin (GA) signalling pathways [15-18]. A recent study has demonstrated that the wrky12 A. thaliana mutants underwent secondary thickening in the walls of certain pith cells that were associated with the ectopic deposition of lignin, xylan and cellulose, leading to an approximately $50 \%$ increase in biomass density in stem tissues. This finding suggests the possibility of significantly increasing the mass of fermentable cell wall components in bioenergy crops [19].

Together, the above findings suggest that the WRKY transcription factors play a crucial role in plant development and disease resistance [6]. Whereas previous research has been largely restricted to Arabidopsis, tobacco and rice, data regarding the WRKY family of transcription factors in cotton (Gossypium hirsutum L.), an important economic crop, are notably limited. In the present study, a cDNA clone, GhWRKY15, encoding a putative IId WRKY gene, was isolated and characterised, and the gene expression patterns under various biotic and abiotic stresses were investigated. We obtained 
information on the potential of overexpression of GhWRKY15 to alter responses to fungal or viral pathogens and developmental processes in tobacco. This study suggests the alteration of responses to disease attacks in GhWRKY15 overexpressing tobacco may be related to multiple defence signalling pathways, and further via regulation of the reactive oxygen species (ROS) network.

\section{Results}

\section{Cloning and characterisation of GhWRKY15}

Due to the vital function of the WRKY motif in the activity of the WRKY family members, we designed a pair of degenerate primers targeting this region (DP1 and DP2, see Additional file 1: Table S2) in an attempt to isolate WRKY family members from cotton. Using this primer pair, the internal conserved region of one IId WRKY subfamily member was obtained. Next, rapid amplification of cDNA ends PCR (RACE-PCR) was used to identify the full-length cDNA, and a 419-bp 5' untranslated region (UTR) and a 546-bp 3' UTR were amplified. To verify the full-length cDNA sequence, two specific primers were designed, and sequence analysis revealed that the full-length cDNA contained a 942-bp open reading frame (ORF) encoding a 314-amino acid protein with a predicted molecular mass of $34.872 \mathrm{kDa}$ and an isoelectric point of 10.07. Because this putative cotton WRKY family member shares a high degree of homology with WRKY15 in A. thaliana, we designated this gene GhWRKY15 (GenBank: GU207867).

A protein sequence comparison between GhWRKY15 and the other plant WRKY proteins demonstrated that the amino acid sequences of these proteins are highly similar, with a homology of $42.60 \%$ to NtWRKY3 (BAA77358), 49.70\% to AtWRKY15 (NP_179913), 54.27\% to StWRKY2 (ABU49721), and 63.19\% to PtWRKY16 (ACV92028) (Figure 1A). The predicted GhWRKY15 protein contains an approximately 60-amino acid WRKY domain that is composed of the conserved amino acid sequence (WRKYGQK) and a zinc-finger motif (C-X $\mathrm{X}_{4-5}-\mathrm{C}$ $\left.\mathrm{X}_{22-23}-\mathrm{H}-\mathrm{X}_{1}-\mathrm{H}\right)$, and a short conserved structural motif (HARF), indicating that GhWRKY15 belongs to Group IId of the WRKY family [20]. Additionally, a putative nuclear localisation signal (NLS), KKRK, was found at position 220-223 (Figure 1A).

A phylogenetic analysis was performed to determine the evolutionary relationship between GhWRKY15 and the other WRKY proteins (Figure 1B). Overall, WRKY proteins can be classified into three groups, and Group II can be further divided into five subgroups (IIa, IIb, IIc, IId and IIe). The phylogenetic analysis demonstrated that GhWRKY15 was closely related to Group IId WRKY family members, including PtWRKY26, StWRKY2, BnWRKY7, AtWRKY7 and AtWRKY15. These results strongly suggest that GhWRKY15 belongs to Group IId of the WRKY family, the members of which may share some similar functions in different species.

To examine the genomic organisation of the GhWRKY15 locus, we amplified the GhWRKY15 genomic sequence with a pair of specific primers that had been designed based on the full-length cDNA sequence. The full-length GhWRKY15 genomic sequence was 1306 bp in length, and two short introns (115 bp and $90 \mathrm{bp}$ ) that exhibited a high AT content and had a typical 5'-GT splice donor and 3'-AG splice acceptor were detected. The comparative analysis of the GhWRKY15 (GenBank: GU207869), AtWRKY7 (NC_003075), AtWRKY15 (NC_003071) and VvWRKY (NW_002239918) genomic sequences revealed that the number and position of the introns were wellconserved (Additional file 2: Figure S1). Specifically, the insert position of the second intron, called an R-type intron, was in the arginine codon of the WRKY domain. Additionally, we also determined the copy number of the GhWRKY15 locus in cotton using fluorescence real-time quantitative PCR (qPCR) analysis with RNA-dependent RNA polymerase 6 (GhRDR6), which is present in the cotton genome as single copy [21], as an internal standard. The standard curve of GhRDR6 and GhWRKY15 is shown in Additional file 3 (Figure S2), and the correlation coefficients between the standard curves of GhRDR6 and GhWRKY15 were highly significant. As shown in Additional file 1 (Tables S3, S4), our results demonstrate that GhWRKY15 is probably a single-copy gene in cotton.

\section{GhWRKY15 is localised to the nucleus}

To investigate the mechanism underlying the regulatory activity of GhWRKY15 in development and during the stress response, the subcellular localisation of GhWRKY15 was predicted using the PSORT programme. An NLS (KKRK) was identified in GhWRKY15, suggesting that it is mostly located in the nucleus. To confirm this prediction, a biolistic transformation system was used for a transient assay, and two constructs, 35SGhWRKY15::GFP and 35S-GFP (Figure 2A), were individually introduced into onion epidermal cells. The fluorescence was observed using a confocal laser scanning microscope with DAPI staining to detect the nuclei and interference contrast images to detect the whole-cell structures. As shown in Figure 2B, the fluorescence was predominantly localised to the nucleus in the onion epidermal cells expressing 35SGhWRKY15::GFP, whereas the fluorescence was present throughout the cytoplasm and nucleus in the cells transfected with the control 35S-GFP construct. These results indicate that the GhWRKY15 protein is located in the nucleus, and this localisation may aid in the determination of both the function and the molecular mechanism underlying the function of this protein. 
(A)

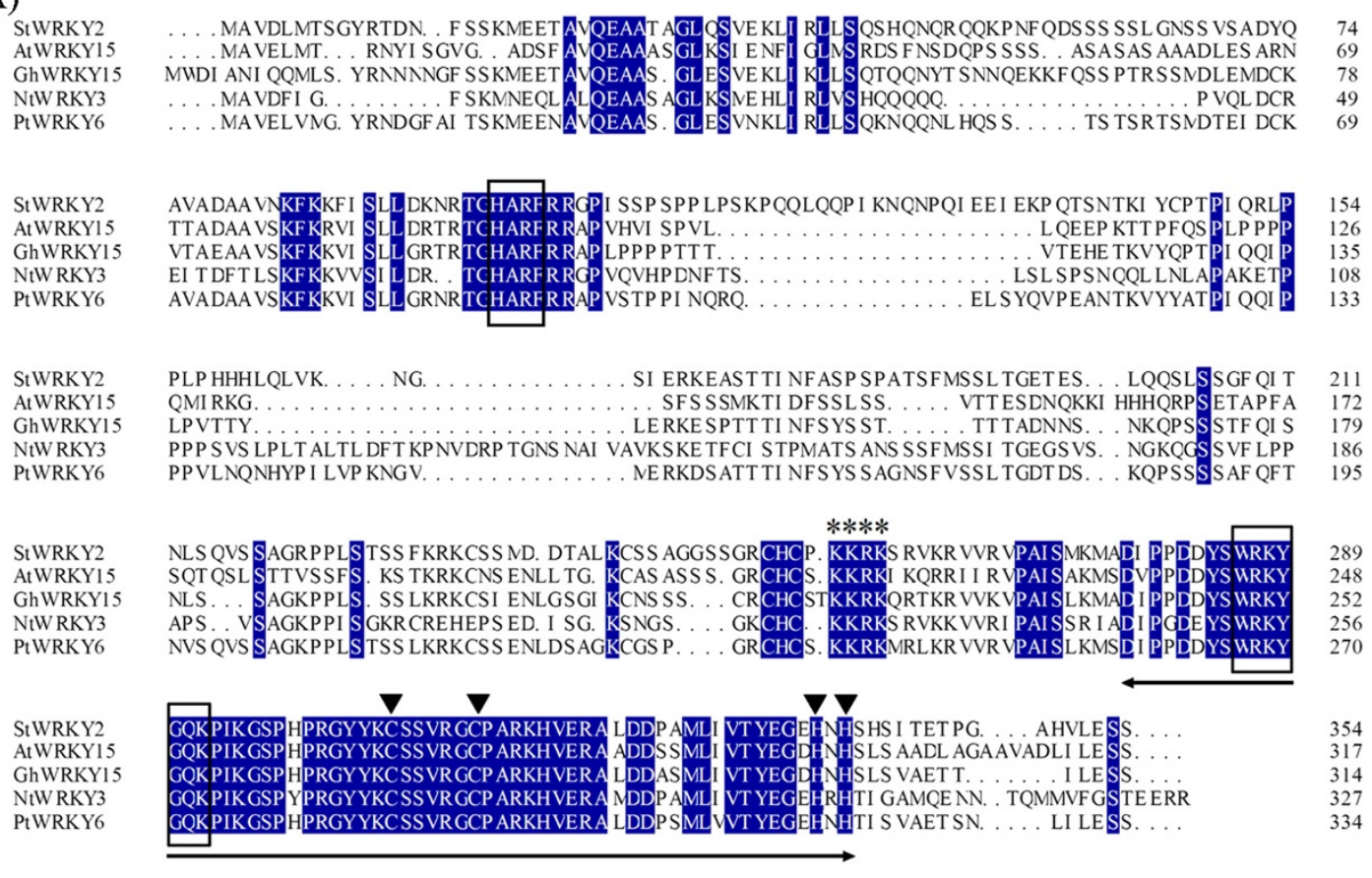

(B)

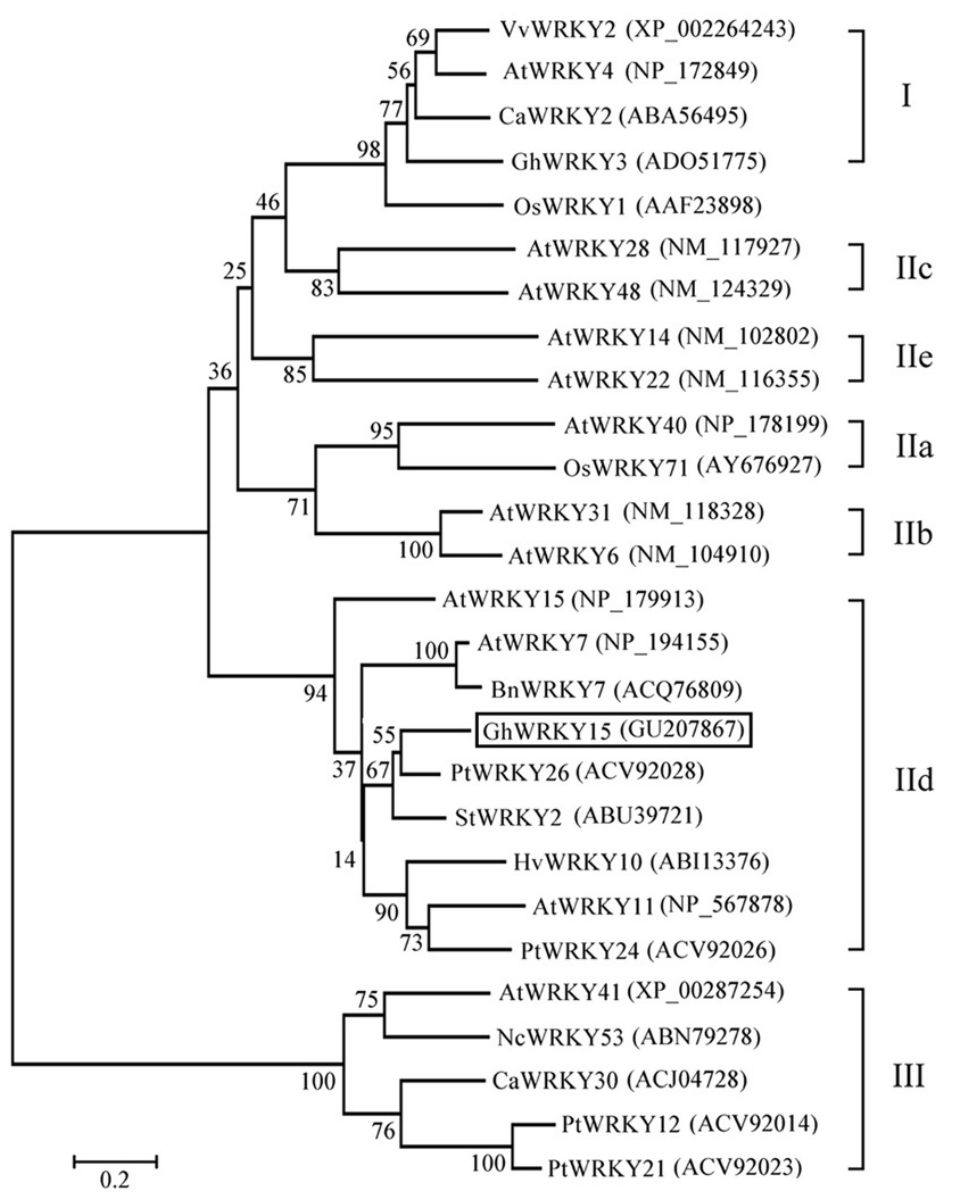

Figure 1 (See legend on next page.) 
(See figure on previous page.)

Figure 1 Characterisation of WRKY transcription factors from various species. (A) Identical amino acids are highlighted in blue. The approximately 60-amino acid WRKY domain and the $\mathrm{C}$ and $\mathrm{H}$ residues in the zinc-finger motif $\left(\mathrm{C}-\mathrm{X}_{4-5}-\mathrm{C}-\mathrm{X}_{22-23}-\mathrm{H}-\mathrm{X}_{1}-\mathrm{H}\right)$ are marked by the two-headed arrow and triangle, respectively. The short conserved HARF structural motif and the highly conserved amino acid sequence WRKYGQK in the WRKY domain are boxed. (B) Phylogenetic analysis of GhWRKY15 in relation to other plant WRKY transcription factors. The WRKY transcription factors used are as follows: GhWRKY15 (GU207867) and GhWRKY3 (ADO51775) from G. hirsutum, PtWRKY26 (ACV92028), PtWRKY24 (ACV92026), PtWRKY12 (ACV92014) and PtWRKY21 (ACV92023) from P. tomentosa, StWRKY2 (ABU49721) from S. tuberosum, AtWRKY15 (NP_179913), AtWRKY7 (NP_194155), AtWRKY11 (NP_567878), AtWRKY4 (NP_172849), AtWRKY40 (NP_178199), AtWRKY31 (NM_118328), AtWRKY6 (NM_104910), AtWRKY28 (NM_117927), AtWRKY48 (NM_124329), AtWRKY14 (NM_102802), AtWRKY22 (NM_116355), and AtWRKY41 (XP_00287254) from A. thaliana, BnWRKY7 (ACQ76809) from Brassi canapus, CaWRKY2 (ABA56495) and CaWRKY30 (ACJ04728) from Capsicum annuum, HvWRKY10 (ABI13376) from Hordeum vulgare, VvWRKY2 (XP_002264243) from Vitis vinifera, OsWRKY71(AY676927) and OsWRKY1 (AAF23898) from O. sativa and NcWRKY53(ABN79278) from Noccaea caerulescens.

\section{Analysis of partial putative cis-acting elements in the GhWRKY15 promoter}

A 1012-bp fragment of the GhWRKY15 promoter (GenBank: GU207869) was obtained using Inverse PCR (I-PCR) and nested PCR. An analysis of this region using the PlantCARE databases revealed a group of putative cis-acting elements that suggest that GhWRKY15 may play a role in the response to environmental stress. Specifically, we identified pathogen/elicitor-related elements including RAV1AAT, ARE, W-box and TL1ATSAR, abiotic stress responsive element including ACGTATERD1, HSE, LTR and MBS and tissue-specific and development-related elements including a Skn-1 motif, circadian, OSE2ROOTNODULE, POLLEN1LELAT52 and AACACOREOSGLUB1. All of the identified cis-elements are listed in Table 1.

\section{GhWRKY15 expression in cotton following exposure to diverse biotic stresses, SA, methyl jasmonate (MeJA) and ET}

To examine the role of GhWRKY15 in response to biotic stress in cotton, cotton seedlings were infected with three different fungi (C. gossypii, Fusarium oxysporum f. sp. vasinfectum and Rhizoctonia solani) using the root dip method. As shown in Figure 3A, B, C and Additional file 4 Figure S3A, B, C, these pathogen treatments dramatically enhanced the accumulation of GhWRKY15 transcripts. The maximum accumulation of the GhWRKY15 transcripts occurred 2, 3 and $5 \mathrm{~d}$ after treatment with these three fungi, respectively. There was no obvious change in the expression of GhWRKY15 under normal conditions without any pathogen infection within 7 days. These data suggest that GhWRKY15 may play important roles in the response to pathogens.

To elucidate the molecular mechanism underlying the responsiveness to various biotic stresses, we examined the expression of GhWRKY15 following treatment with exogenously applied SA, MeJA and ET, which are all phytohormones involved in different signalling pathways. As shown in Figure 3D, E, F, and Additional file 4 (Figure S3D, E, F), the expression of GhWRKY15 was obviously enhanced by SA, MeJA (an analogue of JA) and ET. After the SA treatment, GhWRKY15 expression was noticeably enhanced at $8 \mathrm{~h}$. Following the MeJA treatment, transcripts of GhWRKY15 accumulated at $4 \mathrm{~h}$ and reached a peak from $8 \mathrm{~h}$ to $24 \mathrm{~h}$. Under ET treatment, GhWRKY15 accumulated at $2 \mathrm{~h}$, and reached a peak at $4 \mathrm{~h}$. But ET treatment does not lead to a higher expression than SA or MeJA treatment, respectively. Therefore, GhWRKY15 may be involved in the SA, ET and JA signalling pathways that function in disease resistance.

\section{Tobacco plants overexpressing GhWRKY15 exhibit enhanced viral and fungal resistance}

To investigate the effects of constitutive overexpression of GhWRKY15 on tobacco, full-length GhWRKY15 was cloned into the binary vector pBI121 by replacing the GUS gene, and tobacco lines overexpressing GhWRKY15 were generated. A total of 14 independent transgenic lines were obtained following selection with kanamycin, and these lines were confirmed using PCR. Additionally, 10 lines of transgenic $T_{1}$ plants were randomly selected for an expression analysis. The expression of the transformed GhWRKY15 gene in the leaf tissue was detected using northern blots, whereas the control, wild-type (WT) tobacco lines demonstrated no expression (Figure 4A). Three independent lines, OE1 (6\#), OE2 (9\#) and OE3 (4\#), which exhibited different expression levels of the target gene were used for further functional analysis.

The disease resistance tests were performed on the $T_{2}$ progeny of the three transgenic lines using two kinds of viruses and fungi and compared with the resistance of WT plants. Six-week-old transgenic tobacco plants were inoculated with tobacco mosaic virus (TMV) or cucumber mosaic virus (CMV). Minor disease symptoms were observed in the WT plants seven days after inoculation with TMV, with the leaf veins and adjacent tissue colour fading and appearing dark green, whereas no symptoms were seen in any of the three transgenic lines. The leaves of the TMV-inoculated lines 10 days after inoculation are shown in Figure 4B. However, there were no significant differences in the disease symptoms between the 


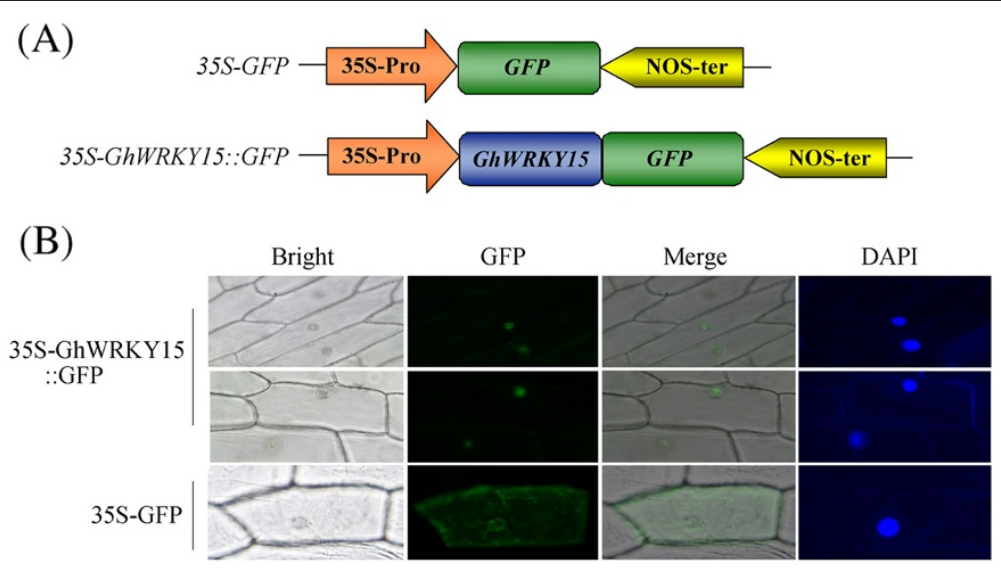

Figure 2 Subcellular localisation of the GhWRKY15::GFP fusion protein. (A) Schematic representation of the 35 S-GhWRKY15::GFP fusion construct and the 35 S-GFP construct. GFP was fused in frame to the C terminus of GhWRKY15. (B) Onion epidermal cells transiently expressing either the 35 S-GhWRKY15::GFP and 35 S-GFP construct were viewed using a confocal laser scanning microscope. The nuclei of the onion cells were visualised using DAPI staining.

transgenic and WT plants fourteen days after inoculation with TMV; both plants exhibited severely diseased, crinkled leaves, leaf distortion, plant dwarfism and slow growth. For the CMV inoculation, no difference in the disease symptoms was observed between the WT and transgenic lines up to seven days after the inoculation, but the WT plants exhibited significantly more severe disease symptoms relative to the transgenic plants after fourteen days (Figure 4B). Furthermore, the expression level of the coat proteins $(\mathrm{CP})$ gene was much lower in both the TMV- and CMV-inoculated transgenic plants than in the WT line (Figure 4C). Additionally, as detected using ELISAs, the average expression of the TMV and CMV CPs in the transgenic lines was lower than the expression in the WT line (Figure 4D).

To investigate whether GhWRKY15-overexpressing tobacco plants possess enhanced fungal resistance compared with WT plants, conidial suspensions of Colletotrichum gossypii and Phytophthora parasitica spores were sprayed on detached leaves. Visually, the detached leaves of the transgenic plants exhibited more resistance to both C. gossypii and P. parasitica infection than the WT plants (Figure 5A). To quantify the lesions, the number and diameter of the disease spots in the wildtype and transgenic plants were measured, and the data were consistent with what was observed visually (Figure 5B, C).

Overexpression of GhWRKY15 affects the expression of PR genes and ethylene (ET) biosynthesis-related genes

Because many WRKY transcription factors have been shown to be involved in activating the transcription of defence genes, particularly the transcription of PR genes [3], the expression of PR genes was investigated using qPCR after the plants were infected with TMV or $C$. gossypii. Following infection with TMV, the expression of PR genes, including PR1, PR2 ( $\beta$-1,3-glucanase), PR4 and $P R 5$ (osmotin), was greater in the three transgenic lines than the expression in the WT plants (Figure 6A). Moreover, the expression of the SA signalling-related gene NPR1 $[3,22]$ was also slightly enhanced. To further elucidate the possible mechanisms underlying the enhanced viral resistance of the transgenic plants, we examined the expression of two enzymes that are involved in ET biosynthesis, 1-aminocyclopropane-1carboxylic acid (ACC) synthase (ACS), which catalyses the conversion of $S$-adenosyl-L-Met into ACC, and ACC oxidase (ACO), which converts ACC into ET [23]. In all three transgenic lines, the expression levels of ACO and ACS were higher than those in WT plants infected with TMV (Figure 6A). Similar results were obtained following C. gossypii infection, except that the transcription of PR5 was inhibited in the transgenic plants compared with the WT plants (Figure 6B). In addition, the expression of the disease-responsive gene SAR8.2 $l$ was significantly upregulated (Figure 6B).

\section{Overexpression of GhWRKY15 decreases the accumulation} of ROS and activates the expression of oxidation-related genes

Some virus-host plant interactions result in oxidative stress and the generation of ROS [24] $\mathrm{H}_{2} \mathrm{O}_{2}$, singlet oxygen, $\mathrm{O}_{2}^{-}$, and hydroxyl radicals are all ROS, and we monitored the accumulation of $\mathrm{H}_{2} \mathrm{O}_{2}$ in transgenic plants and WT plants following exposure to TMV, CMV or C. gossypii. The accumulation of $\mathrm{H}_{2} \mathrm{O}_{2}$ was detected using histochemical analyses via $\mathrm{DAB}$ staining. As seen by the visible accumulation of a brown precipitate, the leaves of transgenic tobacco plants accumulated lower levels of $\mathrm{H}_{2} \mathrm{O}_{2}$ relative to the WT 
Table 1 Putative cis-acting elements of the promoter of GhWRKY15

\begin{tabular}{|c|c|c|}
\hline Cis-element & Position & Sequence \\
\hline \multicolumn{3}{|c|}{ Light responsive elements } \\
\hline AE-box & $-445(-)$ & AGAAACTA \\
\hline ATCC-motif & $-575(+)$ & CAATCCTC \\
\hline Box 4 & $-362(+),-1013(+)$ & ATTAAT \\
\hline G-box & $-752(+)$ & GACATGTGGT \\
\hline I-box & $-541(+)$ & GATATGG \\
\hline LAMP-element & $-722(+)$ & CTTTATCA \\
\hline Sp1 & $-4(+),-31(-)$ & $C C(G / A) C C C$ \\
\hline TCT-motif & $-444(-)$ & TCTTAC \\
\hline \multicolumn{3}{|c|}{ Tissue-specific and development-related elements } \\
\hline Skn-1_motif & $-522(+),-628(-)$ & GTCAT \\
\hline circadian & $-350(+),-763(-)$ & CAANNNNATC \\
\hline OSE2ROOTNODULE & $-808(+)$ & CTCTT \\
\hline POLLEN1LELAT52 & $-82(+),-163(+),-306(+),-345(+)$ & AGAAA \\
\hline AACACOREOSGLUB1 & $-17(+)$ & AACAAAC \\
\hline \multicolumn{3}{|c|}{ Pathogen/elicitor-related elements } \\
\hline RAV1AAT & $-197(+),-256(+),-320(+),-973(+)$ & CAACA \\
\hline ARE & $-534(+),-705(+)$ & TGGTTT \\
\hline W-box & $-867(+)$ & $(\mathrm{T})(\mathrm{T}) \mathrm{TGAC}(\mathrm{C} / \mathrm{T})$ \\
\hline TL1ATSAR & $-855(+)$ & TTCTTCTTCAG \\
\hline \multicolumn{3}{|c|}{ Abiotic stress responsive element } \\
\hline ACGTATERD1 & $-118(+)$ & ACGT \\
\hline HSE & $-301(+),-609(+)$ & $\mathrm{A}(\mathrm{A} / \mathrm{G}) \mathrm{AAAATT}(\mathrm{T} / \mathrm{C})(\mathrm{C} / \mathrm{G})$ \\
\hline LTR & $-465(-),-632(+)$ & CCGAAA \\
\hline MBS & $-295(-),-908(+)$ & TAACTG \\
\hline
\end{tabular}

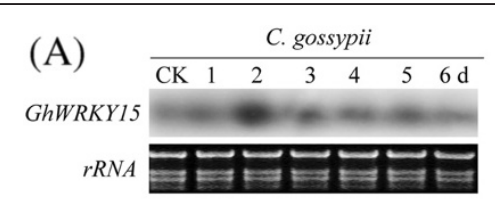

(C)

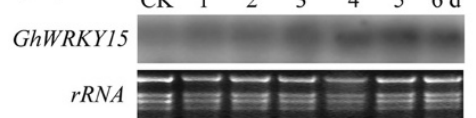

(E)

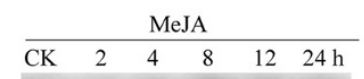

GhWRKY15

rRNA

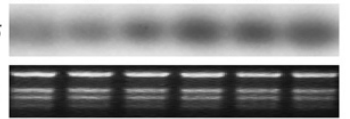

(B)

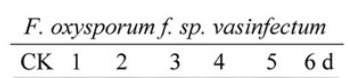

GhWRKY15

$r R N A$

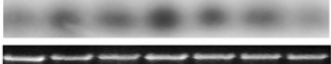

(D)
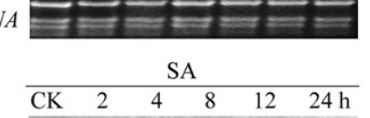

GhWRKY15

$r R N A$

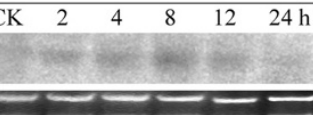

(F)

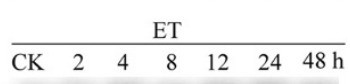

GhWRKY15

rRNA

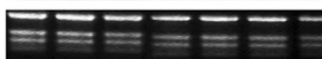

Figure 3 Expression of GhWRKY15 in response to different fungal infections and hormone treatments. Approximately one-week-old cotton seedlings were used for all treatments. For the fungal inoculation, the roots of the cotton seedlings were dipped into conidial suspensions of C. gossypii (A), F. oxysporum f. sp. vasinfectum (B) or R. solani (C) $\left(10^{5}\right.$ conidia/ml). The signalling molecules used were $2 \mathrm{mM} \mathrm{SA} \mathrm{(D),} 100 \mu \mathrm{MM}$ MeJA (E) and ET released from $5 \mathrm{mM}$ ethephon (F). Whole seedling plants were collected for RNA extraction. Ethidium bromide-stained rRNA was included as a loading control. 


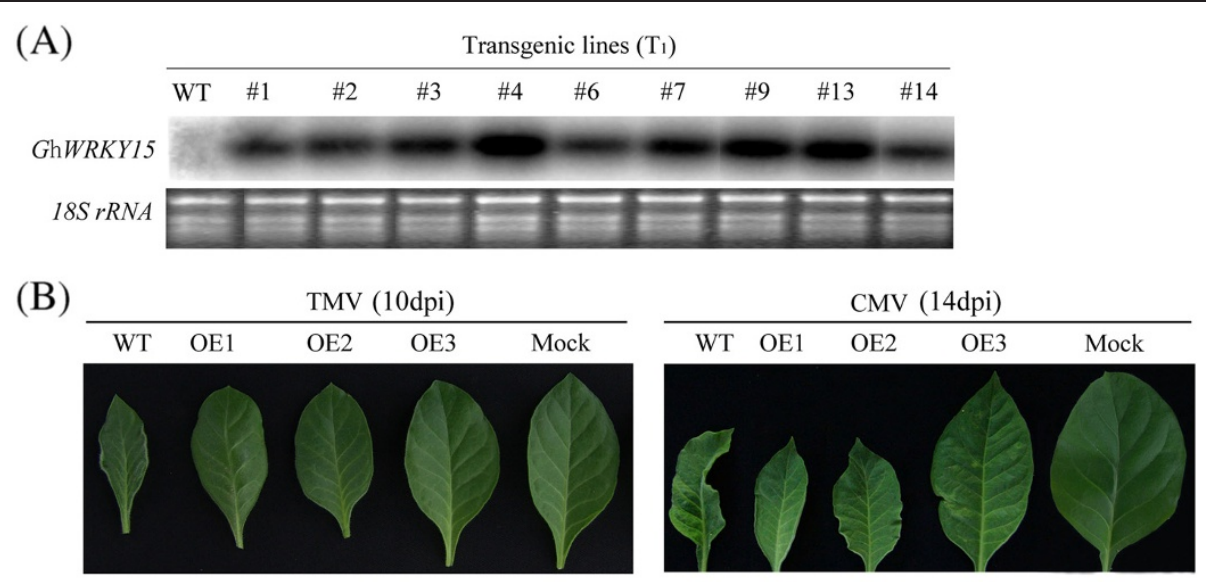

(C)

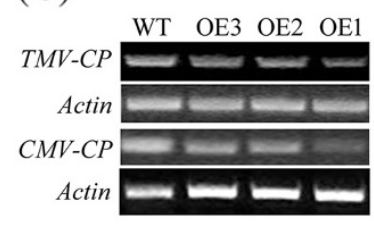

(D)

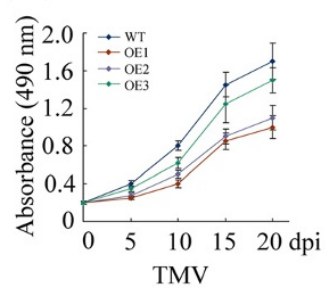

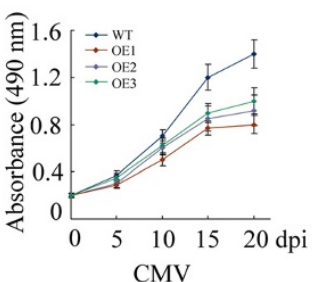

Figure 4 Enhanced resistance of GhWRKY15-overexpressing tobacco to viruses. (A) Northern blot analysis of the expression levels of GhWRKY15 in $T_{1}$ transgenic and WT tobacco under normal conditions. Two leaves were tested for the GhWRKY15 transgenic tobacco. WT: wildtype. (B) Leaf symptoms of tobacco plants infected with TMV (10 days post-inoculation) or CMV (14 days post-inoculation). OE: GhWRKY15overexpressing tobacco; Mock: mock inoculation; CP: coat proteins. (C) RT-PCR analysis of the expression levels of the CP gene in infected transgenic lines (OE1, OE2 and OE3) and the WT line. (D) TMV and CMV titres in the transgenic lines and the wild-type lines. The data are presented as the mean \pm standard error from three independent experiments.

plants (Figure 7A, B, C). A microscopic analysis of the plants following viral infection also indicated less brown precipitate on the leaves of transgenic plants when compared with WT plants (Figure 7A, B), which is consistent with the results defined visually.

A potential redox mediator, methyl viologen (MV), was used to verify whether GhWRKY15 is responsive to oxidative stress. The expression of GhWRKY15 was upregulated $2 \mathrm{~h}$ after treatment with $0.5 \mathrm{mM} \mathrm{MV}$, and it was significantly enhanced at $4 \mathrm{~h}$ (Figure 7D and Additional file 5: Figure S4). To explore the underlying mechanism of the reduced ROS levels in the transgenic plants during the disease resistance process, the expression of six genes was analysed in response to oxidative stress. The enzyme SOD acts as the first line of defence against ROS by transforming $\mathrm{O}^{2-}$ into $\mathrm{H}_{2} \mathrm{O}_{2}$, and such enzymes as APX, CAT and GPX play important roles in regulating intracellular $\mathrm{H}_{2} \mathrm{O}_{2}$ levels [25,26]. To our surprise, with the exception of the upregulation of $N t G P X$, the expression of the other five antioxidant genes (NtSOD, NtAPX1, $N t A P X 2, N t C A T 1$ and $N t C A$ ) was decreased in the transgenic plants relative to the WT plants under normal conditions (Figure 8A). However, when treated with MV, the expression of NtAPX1 and NtGPX was enhanced (Figure $8 \mathrm{~B}$ ) in the transgenic plants compared with the wild-type plants, whereas the NtCAT1 and NtCA expression levels were still decreased in the transgenic plants relative to the wild-type plants. We observed no significant differences in the expression of NtSOD and NtAPX2 between the transgenic and WT lines. These results suggest that the overexpression of GhWRKY15 indeed affected the antioxidant system in tobacco, which led us to further investigate how GhWRKY15 regulated the ROS scavenging pathway. Thus, we examined the enzymatic activities of SOD, POD, CAT and APX in the oxidative response during treatment with TMV or CMV (Figure 9A, B). Compared to the activities observed in WT plants, the activities of POD and APX were significantly increased in the OE lines, but there was a slight decrease in CAT activity. Interestingly, the activity of SOD was significantly increased following infection with TMV, whereas the activity of SOD following infection with CMV was decreased. These results indicate that the role of GhWRKY15 in the ROS-scavenging pathway is complicated. 


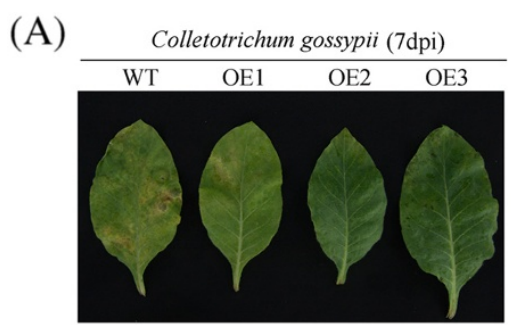

(B)

(C)
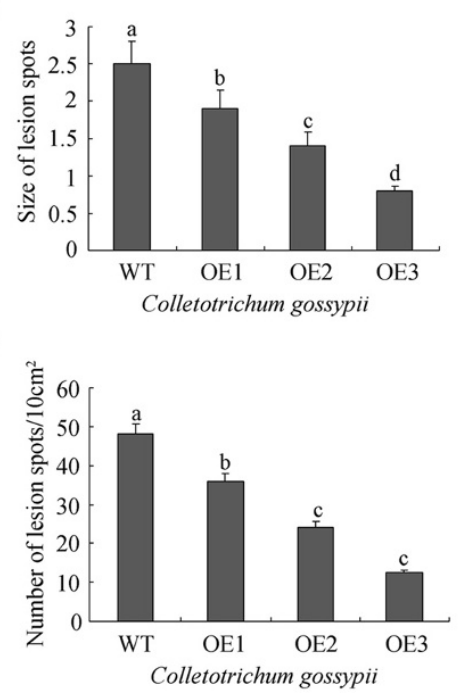
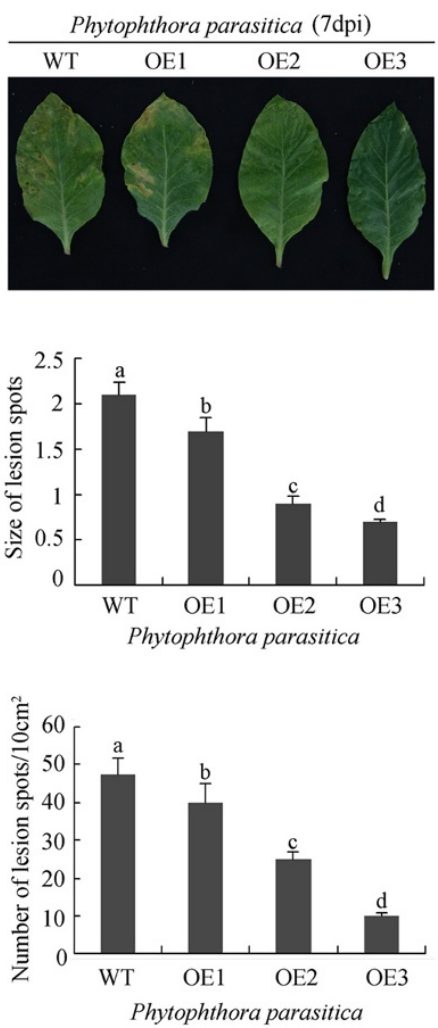

Figure 5 Enhanced resistance of GhWRKY15-overexpressing tobacco to fungi. (A) Leaf symptoms of tobacco plants infected with fungi. The detached leaves in transgenic and wild-type tobacco were inoculated with C. gossypii or P. parasitica suspensions ( $10^{6}$ conidia/ml) prepared in $1 \%$ glucose, and the leaves were photographed 7 days after inoculation. (B) The diameters of the lesions on the inoculated leaves. The diameters of the lesion spots were recorded using the following scoring system: $0,<1 \mathrm{~mm} ; 1,1-2 \mathrm{~mm} ; 2,>2 \mathrm{~mm}$. (C) The numbers of lesions on the inoculated leaves. The number of lesions per $10 \mathrm{~cm}^{2}$ was counted on the inoculated leaves of three independent transgenic and wild-type plants. The values indicated by the different letters are significantly different at $P<0.05$, as determined using Duncan's multiple range tests.

\section{Expression of GhWRKY15 affects plant growth and development}

Many WRKY genes have been reported to affect plant development and growth throughout the entire development and growth period. To investigate the effects of constitutive overexpression of GhWRKY15 on plant growth and development in tobacco, the growth phenotype of both WT plants and transgenic lines was monitored. Except for the later germination of the transgenic plants relative to the WT plants (Figure 10A), we observed no difference between the plants until approximately ten weeks. The transgenic plants entered the shooting stages earlier than did the wild-type plants. As shown in Figure 10B, the stems of the OE plants began to elongate at approximately ten weeks, but the stems of the WT tobacco had no evident elongation. Interestingly, the stems of the transgenic plants were much thinner than the stems of the WT plants during the shooting stage (Figure 11E). Microscopic analyses revealed that the cortical cells of the transgenic plants had smaller diameters relative to the cells of the WT plants (Figure 11C), but no difference in the cell diameter of the pith was observed (Figure 11D). Moreover, no difference was observed in the length of the cells in the WT and transgenic plants (Figure 11B). After the shooting stage of the transgenic plants began, overexpression of GhWRKY15 resulted in higher transgenic plants relative to wild-type plants (Figure 10C). However, this difference in the stem length disappeared at the flower stage. Moreover, GhWRKY15-overexpressing transgenic plants displayed premature flowering by two to three weeks relative to the wild-type plants under normal growth conditions (Figure 10D). Additionally, the bottom leaves of the OE lines exhibited senescence earlier than the bottom leaves of the WT plants (Figure 10E).

Tissue-specific expression of GhWRKY15 and the effects of abiotic stresses on GhWRKY15 expression

To characterise the expression of GhWRKY15 under abiotic stresses, including cold, high salinity, wounding and drought (PEG6000), northern blots were performed 


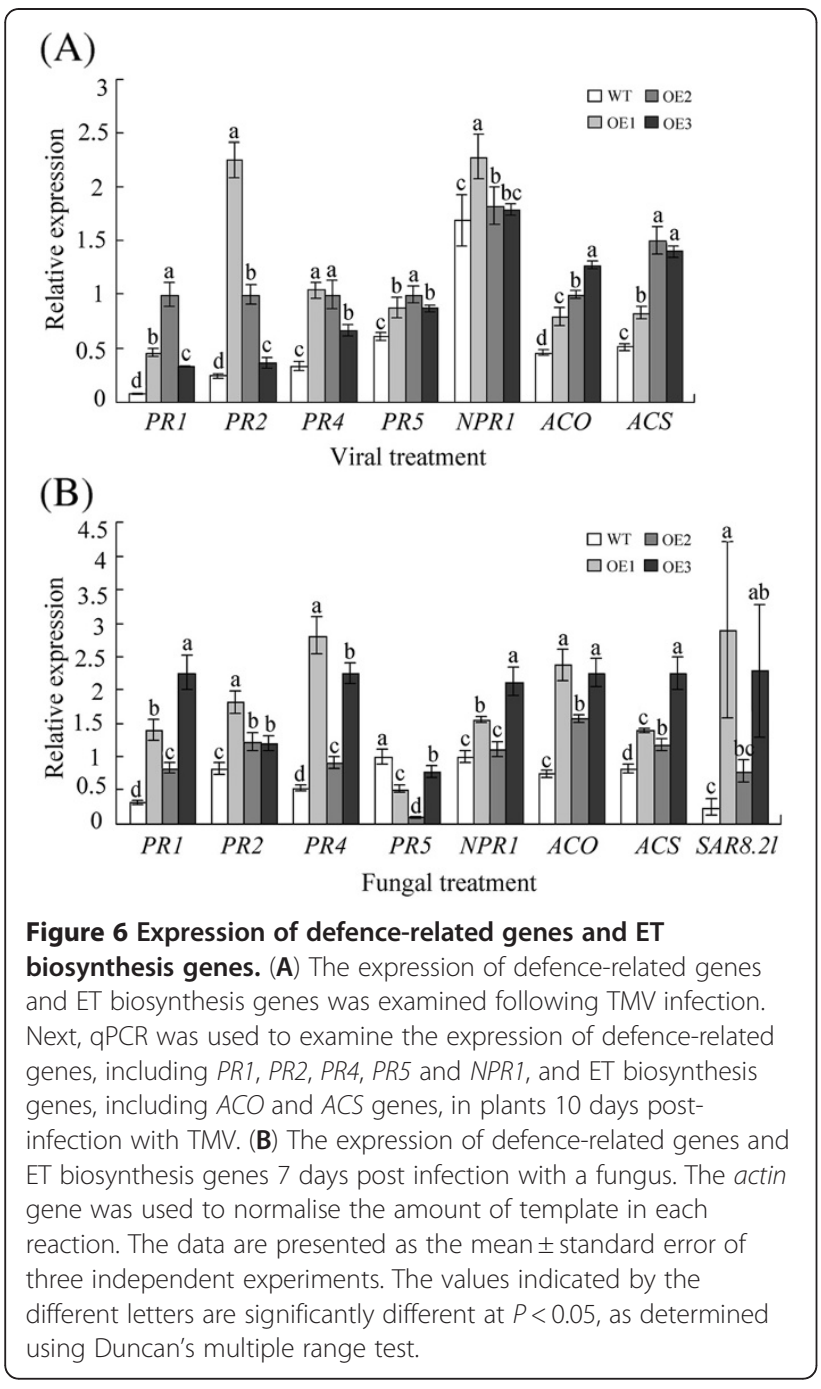

using total RNA extracted from cotton seedlings. Whereas the expression of GhWRKY15 exhibited negligible changes following $\mathrm{NaCl}$ treatment (Figure 12C), the other three treatments resulted in a dramatic increase in the expression of GhWRKY15 within $8 \mathrm{~h}$. As shown in Figure 12B, D, E, the expression patterns were different. GhWRKY15 expression increased sharply within $2 \mathrm{~h}$ following wounding, and the high level due to the wounding was present until $8 \mathrm{~h}$. Following the cold treatment, increased GhWRKY15 expression was detected at $2 \mathrm{~h}$, and this expression continued gradually to increase. The peak expression of GhWRKY15 following drought treatment occurred at $8 \mathrm{~h}$, after which the expression level decreased gradually. These results indicate that GhWRKY15 expression can be regulated by abiotic stresses. Intriguingly, there was a greater accumulation of GhWRKY15 transcripts in the root and stem than in the leaves (Figure 12A), and $\mathrm{GA}_{3}$ treatment led to a higher expression level from $4 \mathrm{~h}$ to $24 \mathrm{~h}$ (Figure 12F), whereas ABA did not increase expression until $24 \mathrm{~h}$ (Figure 12G). These results suggest that GhWRKY15 may play a role not only in response to abiotic stresses but also in plant growth and development.

\section{Discussion}

Although five major families of plant transcription factors, including bZIP, WRKY, MYB, EREBF and homeodomain protein, have been shown to play roles in the regulation of the plant defence response [2], little is known about the exact function or mechanism of individual transcription factors. Generally, it is believed that the WRKY family of transcription factors plays major roles in plant responses to biotic and abiotic stresses and during development $[3,10,11,13,19]$. Most functional analyses of WRKY transcription factors have been restricted to $A$. thaliana and $O$. sativa, and few WRKY family members from cotton have been reported previously. To explore the function of the WRKY transcription factors in cotton, we isolated a WRKY gene from $G$. hirsutum, which is an important economic crop cultivated worldwide.

Due to the high similarity between the sequence of the protein encoded by the WRKY gene obtained from cotton and those of several WRKYs obtained from N. tabacum, A. thaliana, Solanum tuberosum and Populus tomentosa, we verified that the gene we isolated from cotton is a WRKY transcription factor gene. The phylogenetic tree and the short conserved HARF structural motif further indicated that the GhWRKY gene belongs to subgroup IId, with AtWRKY15, AtWRKY11 and AtWRKY7 as its closest homologues in A. thaliana [20]; As a result, we designated the isolated gene GhWRKY15. Consistent with the putative role of WRKY proteins as transcription factors, a nuclear targeting sequence (KKRK) was identified in the C-terminal region of the protein, and the targeting experiment suggested that GFP-tagged GhWRKY15 was located in nucleus. These results suggest that, similar to GhWRKY3 [27], GhWRKY15 might function in the nucleus.

To investigate the effects of constitutive overexpression of GhWRKY15 on the response to pathogen resistance, GhWRKY15 overexpressing-tobacco plants were inoculated with two types of viruses and two pathogenic fungi. The plants constitutively overexpressing GhWRKY15 were more resistant to TMV and CMV infection compared with the WT tobacco plants. This observation diverges from previous research demonstrating that the virus-induced gene silencing (VIGS) of NbWRKY1, NbWRKY2, or NbWRKY3 suppressed resistance to TMV and that the size of the hypersensitive response (HR) lesions was also reduced in NbWRKY1silenced plants [22]. The GhWRKY15 transgenic plants also exhibited increased resistance to fungal pathogens (C. gossypii and P. parasitica) compared with wild-type 


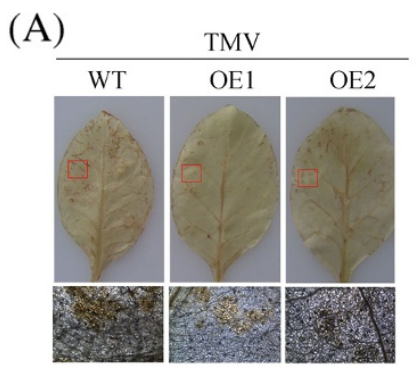

(C)

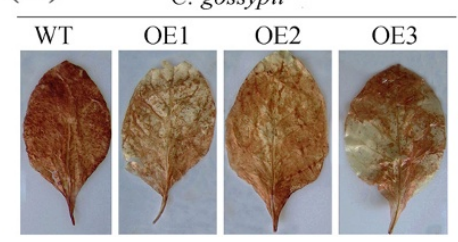

(B)

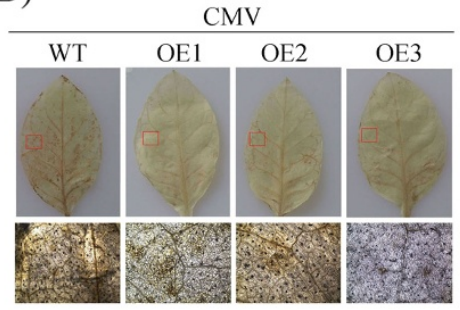

(D)

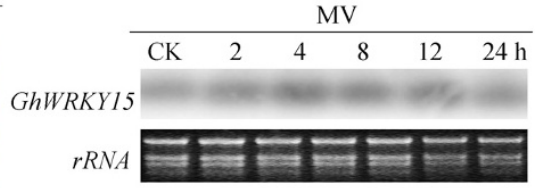

Figure 7 Expression of GhWRKY15 in tobacco decreased the accumulation of ROS, and MV enhanced GhWRKY15 expression. (A), (B) and (C) show that the expression of GhWRKY15 in tobacco decreased the accumulation of ROS after TMV, CMV or C. gossypii treatment, respectively. The level of $\mathrm{H}_{2} \mathrm{O}_{2}$ in the tobacco leaves was determined using $1 \mathrm{mg} / \mathrm{ml} \mathrm{DAB}$ as substrate. The top figure indicates the visualisation of the $\mathrm{H}_{2} \mathrm{O}_{2}$ accumulation, and the bottom figure shows the microscopic observations of the brown precipitate. (D) MV enhances GhWRKY15 expression. Approximately one-week-old cotton seedlings were used for the $0.5 \mathrm{mM}$ MV treatment. Ethidium bromide-stained rRNA was included as a loading control.

plants. Previously, overexpression studies examining a pair of allelic genes OsWRKY45-1 and OsWRKY45-2 demonstrated that these genes positively regulated resistance to the rice fungal pathogen Magnaporthe grisea [28]. Our data presented above indicate that GhWRKY15 overexpression increases the tolerance of the plant to pathogens; therefore, the upregulation of GhWRKY15 in cotton seedlings in response to pathogens, including R. solani, C. gossypii and F. oxysporum, suggests that GhWRKY15 may participate in the regulation of plant responses to disease defence. The increased expression of $P R 1, P R 2$ and PR4 contributed to the increased resistance of transgenic GhWRKY15overexpressing plants to TMV and C. gossypii. Furthermore, previous studies have demonstrated that WRKY DNA binding-proteins can regulate the transcription of a number of defence-related genes, including the wellstudied PR genes, via binding to the W-box in the promoter of these genes [11,29]. It is noted that the expression of the PR5 gene in transgenic plants during C. gossypii infection was down-regulated compared with the infected WT plants, suggesting that the molecular mechanism underlying the activity of GhWRKY15 is fairly complex under different biotic stresses or that GhWRKY15 may be an integrator of several signalling pathways. SA and JA are two well-studied key mediators of plant resistance. In Arabidopsis, more than 49 WRKY genes can be differentially regulated after infection with $P$. syringae or treatment with SA [30]. Infection of GhWRKY15 transgenic plants with TMV and C. gossypii increased the expression of NPR1, which is a key regulator of the SA-dependent defence pathway and SAR. Furthermore, the analysis of the expression of GhWRKY15 in cotton seedlings showed that GhWRKY15 expression can be affected by SA and MeJA, supporting our hypothesis that GhWRKY15 plays a role in resistance to viral and fungal pathogens via SA- and JA-dependent defence pathways. Interestingly, reports of WRKY proteins that function in both SA- and JA-dependent pathways are relatively limited [7]. Moreover, the expression of two key enzymes that are involved in ET biosynthesis (ACO and ACS) is increased in GhWRKY15 transgenic plants following infection with fungi and viruses. And there was significant increase of GhWRKY15 expression in cotton seedlings following ET treatment. This suggests that the increased disease resistance of GhWRKY15 overexpressing tobacco compared to the wide type may partially depend on the expression of ET synthesis related genes. Additionally, GhWRKY15 expression could be increased under various abiotic stress conditions, including low temperature, wounding and drought, and a group of cis-elements related to biotic and abiotic stresses were identified in the GhWRKY15 promoter. Altogether, these data support our hypothesis that the mechanism of response to various environmental stresses, especially viral and fungal infection, in GhWRKY15 overexpressing plants may be related with multiple signalling pathways, including SA, JA or ET signalling pathways.

Mounting evidence suggests that the ROS network is essential for the induction of disease resistance [31]. For instance, mitogen-activated protein kinase (MAPK) cascades members GhMAPK2 and GhMAPK6 are both 


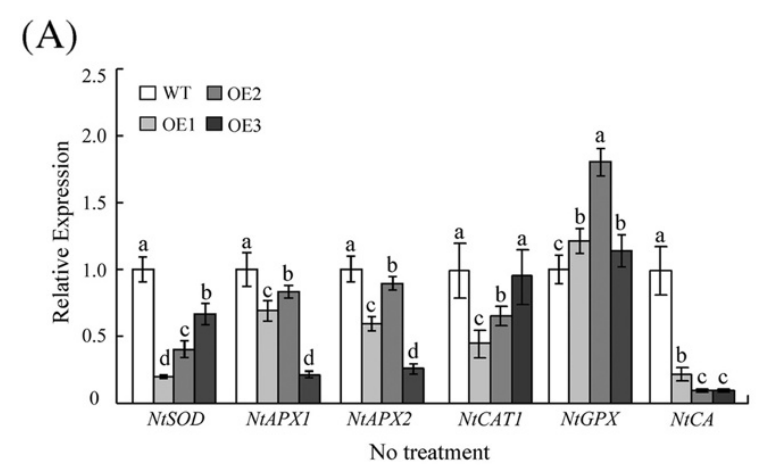

(B)

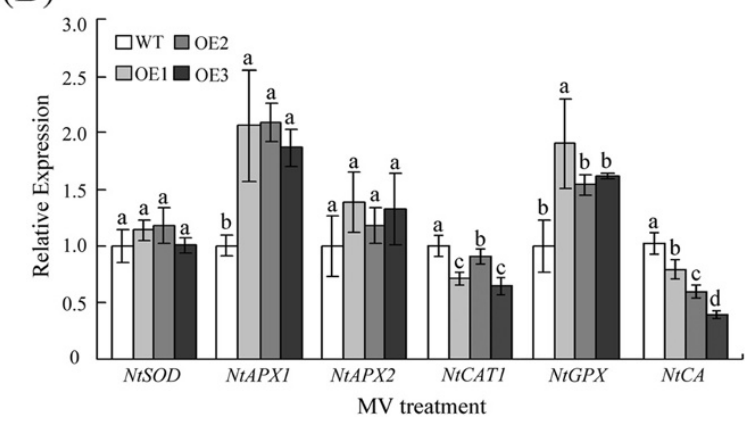

Figure 8 Expression of antioxidant enzymes in transgenic lines. (A) The expression of antioxidant enzymes under normal conditions. (B) The expression of antioxidant enzymes during MV treatment. Also, qPCR analysis was performed to detect the levels of the antioxidant enzymes (NtSOD, NtGPX, NtAPX1, NtAPX2, NtCAT1 and NtCA). Approximately three-week-old transgenic and wild-type tobacco plants were used for the expression analysis. For the MV treatment, the tobacco seedlings were sprayed with $0.5 \mathrm{mM} \mathrm{MV}$ and analyzed $6 \mathrm{~h}$ after treatment. The data are presented as the

mean \pm standard error of three independent experiments. The values indicated by the different letters are significantly different at $P<0.05$, as determined using Duncan's multiple range tests. involved in the response to infection and oxidative stress $[32,33]$. Following challenge with a virus or a fungus, a lower accumulation of $\mathrm{H}_{2} \mathrm{O}_{2}$ and a higher activity of antioxidant enzymes, including POD and APX, were detected in the GhWRKY15-overexpressing plants relative to the WT plants. We reasoned that GhWRKY15 upregulates the activity of POD and APX to increase the oxidative stress tolerance and contributes to the resistance towards biotic stresses in transgenic plants. However, except for $N t G P X$, the expression of the other five antioxidant enzymes examined was reduced in unstressed transgenic seedlings compared with the wild-type plants. These results contrast previous research examining some genes, which appear to play positive roles in the stress response and enhance the expression of most antioxidant enzymes [32,34]. To explain this difference, the expression of the six antioxidant enzymes was examined during treatment with MV, which is a potential redox mediator. Although there was no significant difference in the expression of $N t S O D$ and NtAPX2 in the transgenic plants compared with the WT plants following the treatment with MV, the expression of these genes was increased compared with the expression in plants receiving no treatment. Additionally, the upregulation of $N t G P X$ and NtAPX1 expression during MV treatment suggests that GhWRKY15 overexpression can help plants maintain ROS homoeostasis under oxidative stresses to alleviate injury to the organism. Most recently, it has been reported that there was a substantial increase in the MusaWRKY71 level when banana plantlets were subjected to $\mathrm{H}_{2} \mathrm{O}_{2}$. Moreover, a putative thioredoxin-dependent peroxidase gene belonging to the ROS detoxification system was induced in MusaWRKY71overexpressing cultures [35]. Furthermore, in Arabidopsis, the maximum expression of WRKY53 was detected in
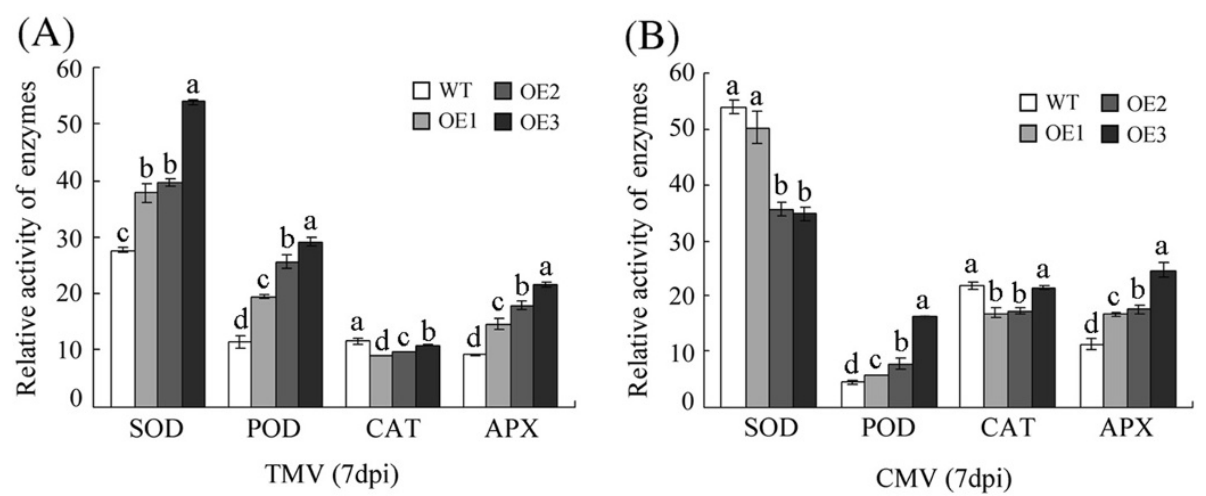

Figure 9 Effect of virus infection on the SOD, POD, CAT and APX activities. (A) and (B) present the SOD, POD, CAT and APX activities 7 days post inoculation with TMV and CMV. The data are presented as the mean \pm standard error of three independent experiments. The values indicated by the different letters are significantly different at $P<0.05$, as determined using Duncan's multiple range tests. 
(A)

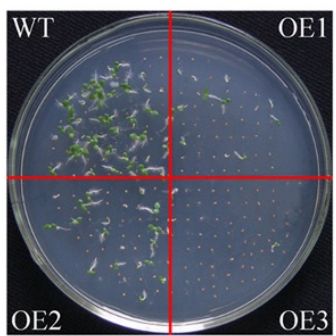

(C)

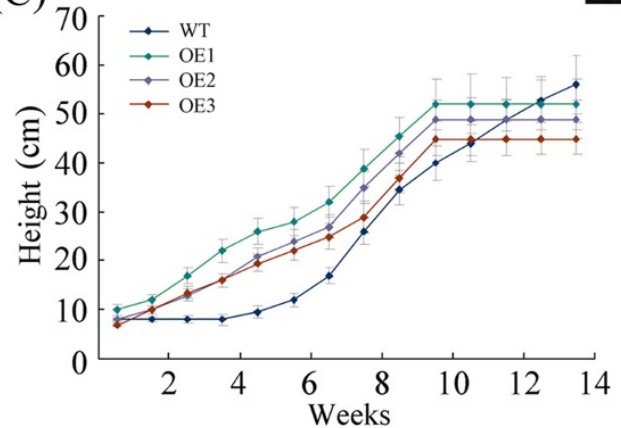

(B)
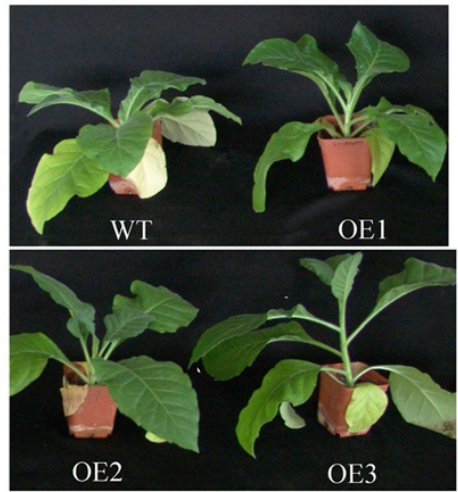

(D) WT $\mathrm{OE}$
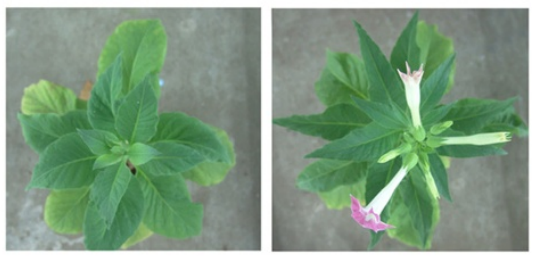

(E)

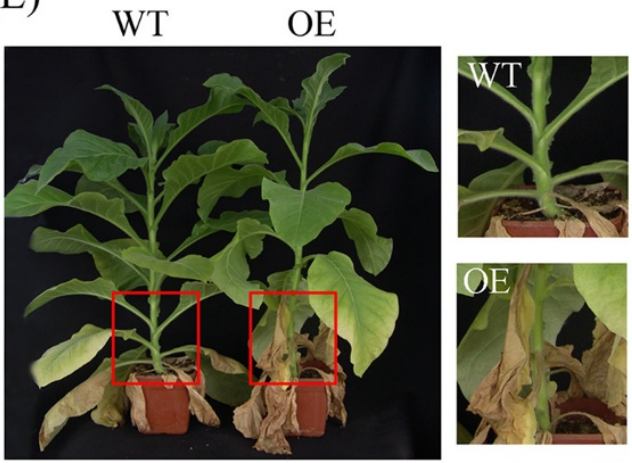

Figure 10 Comparison of the growth and development of the transgenic and wild-type tobacco. (A) Seed germination and growth phenotype of transgenic and wild-type tobacco. (B) The growth phenotype of transgenic and wild-type tobacco at approximately 10 weeks. Differences in stem elongation are clearly observable. (C) The height of transgenic and wild-type tobacco from the shooting stage to the flowering stage. (D) Premature flowering of the transgenic plants relative to the wild-type plants. The growth phenotype was photographed at approximately 22 weeks. (E) The phenotype of the bottom leaves of the transgenic and wild-type tobacco at approximately 18 weeks. The figures are a magnification of the red boxes in (E).

seven-week-old plants overexpressing WRKY53, at which point the $\mathrm{H}_{2} \mathrm{O}_{2}$ level increased significantly [18]. In wildtype cotton seedlings, a substantial increase in the level of GhWRKY15 was also observed following exposure to MV.

Intriguingly, some of the growth and development phenomena observed in plants overexpressing GhWRKY15 are similar to those observed in AtWRKY53 overexpressing plants. Similar to GhWRKY15-overexpressing plants, AtWRKY53-overexpressing plants demonstrated early flowering after sowing, and the senescence of the whole plant with partly necrotic leaves was visible earlier when compared with wild-type plants [18]. Interestingly, overexpression of GhWRKY15 in N. tabacum cv. NC89 promoted stem elongation and inhibited the transversal growth of stems. AtWRKY12 controls cell fate in thickened pith cells by acting as a negative regulator of NST2 and $\mathrm{C} 3 \mathrm{H}$ zinc finger TFs, which, in turn, regulate secondary cell wall synthesis [19]. Although no difference in the diameter and length of pith cells was observed in our 


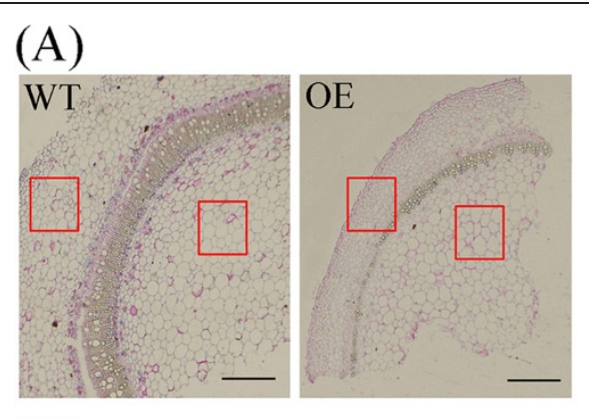

(B)

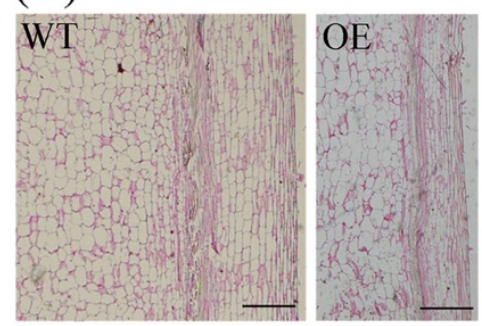

(C)

(D)
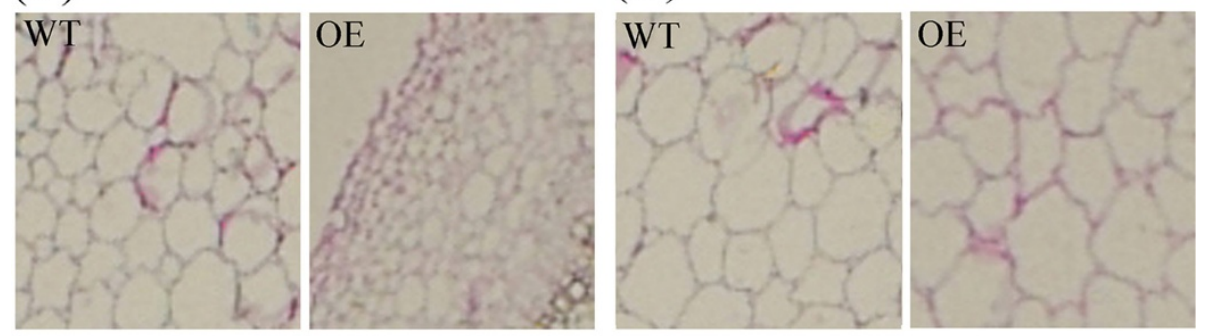

(E)

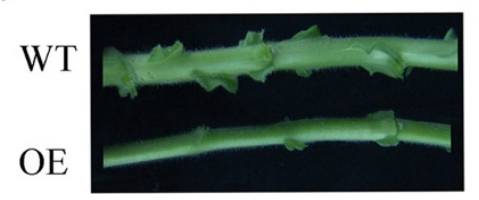

Figure 11 Comparison of stems between transgenic and wild-type tobacco. (A) Transverse section of the stems of transgenic and wild-type tobacco at the shooting stage. (B) Vertical section of the stems of transgenic and wild-type tobacco at the shooting stage. (C) Magnification of the red boxes on the left in (A). (D) Magnification of the red boxes on the right in (A). The left and right red boxes primarily indicate cells of the cortex, vascular bundle and pith. Bar: $100 \mu \mathrm{m}$. (E) Visual differences in the stems of transgenic and wild-type tobacco at the shooting stage.

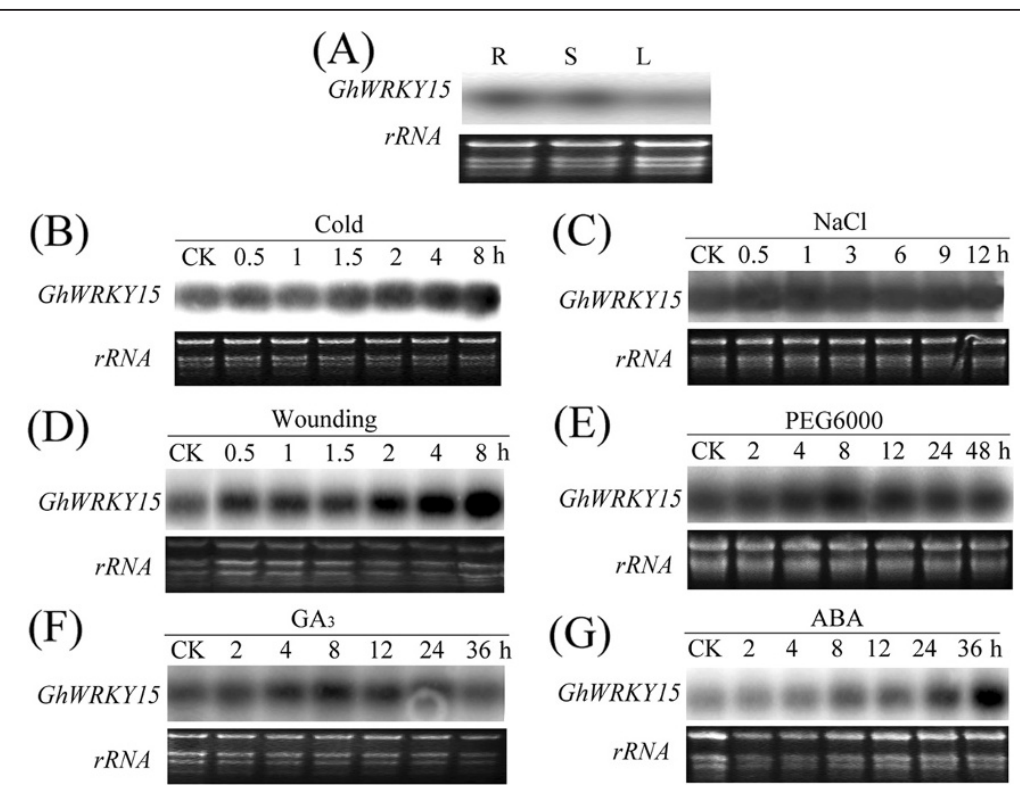

Figure 12 Tissue-specific expression of GhWRKY15 and expression analysis of GhWRKY15 in response to abiotic stresses. Total RNA was extracted from the roots $(R)$, stems $(S)$ and leaves $(L)$ for the tissue-specific expression analysis $(\mathbf{A})$. Total RNA was extracted from the leaves at the indicated time points after treatment with cold $\left(4^{\circ} \mathrm{C}\right)(\mathbf{B}), 200 \mathrm{mM} \mathrm{NaCl}(\mathbf{C})$, wounding (D), $15 \%(\mathrm{~W} / \mathrm{V})$ PEG6000 (E), $500 \mu \mathrm{M} \mathrm{GA}$ (F) or $100 \mu \mathrm{M}$ ABA (G). Ethidium bromide-stained rRNA was included as a loading control. 
transgenic plants, overexpression of GhWRKY15 may affect the fate of cells in the cortex, which had a smaller diameter relative to WT plants. The phytohormone GA is an essential regulator of stem elongation and other developmental processes throughout the life cycle of a plant [36]. The induction of GhWRKY15 expression during $\mathrm{GA}_{3}$ treatment combined with the higher expression of GhWRKY15 in the roots and stems compared with the cotyledon of cotton may clarify the underlying mechanism of the influence of GhWRKY15 overexpression on plant growth and development.

\section{Conclusion}

In conclusion, we identified and characterised a group IId WRKY member in cotton. Our results strongly suggest that overexpression of GhWRKY15 confers tobacco resistance to both viral and fungal pathogens, and indicate that overexpression of GhWRKY15 regulates the stem growth or development in tobacco. The elucidation of the regulatory mechanism of overexpression of GhWRKY15 in tobacco may provide a converging node of regulatory pathways in response to pathogens and oxidative stress and in the regulation of plant development. Although the influence of overexpression of GhWRKY15 in tobacco was explored, the mechanism underlying the function of GhWRKY15 should be elucidated in transgenic cotton and loss-of function data in cotton are particularly needed. Moreover, other research focusing GhWRKY15 is still needed, for example, the study of its interactions with other WRKY proteins or MAPK cascades members.

\section{Methods}

\section{Plant growth and treatments}

Cotton (Gossypium hirsutum L. cv Lumian 22) seedlings were grown in a growth chamber at $28{ }^{\circ} \mathrm{C}$ under a $16 \mathrm{~h}$ light and $8 \mathrm{~h}$ dark photoperiod with a light intensity of $300 \mu \mathrm{mol} \mathrm{m} \mathrm{m}^{-2} \mathrm{~s}^{-1}$. Seven-day-old cotton seedlings were collected for the various treatments. For the tissuespecific expression analysis, the roots, stems and leaves were harvested separately from seven-day-old cotton seedlings. For the hormone treatments, the leaves of uniformly developed seedlings were sprayed with $2 \mathrm{mM} \mathrm{SA}$, $100 \mu \mathrm{M}$ ABA, $100 \mu \mathrm{M}$ methyl jasmonate (MeJA), ET released from $5 \mathrm{mM}$ ethephon, $500 \mu \mathrm{M} \mathrm{GA}_{3}$, or $0.5 \mathrm{mM}$ methyl viologen $(\mathrm{MV})$. For the salt and drought treatments, the seedlings were cultured in solutions containing $200 \mathrm{mM} \mathrm{NaCl}$ or $15 \%$ (w/v) PEG6000, respectively. The mechanical wounding procedure was performed on leaves of seedlings by crushing the leaves with a hemostat, and a low temperature of $4{ }^{\circ} \mathrm{C}$ was used for the cold treatment. For the fungal pathogen treatment, the roots of cotton seedlings were dipped into Fusarium oxysporum f. sp. vasinfectum, Rhizoctonia solani, or $C$. gossypii conidial suspensions $\left(10^{5}\right.$ conidia/ml $)$. The cotyledons, leaves, stems and roots were harvested, frozen directly in liquid nitrogen and stored at $-80{ }^{\circ} \mathrm{C}$ for later use.

\section{Cloning of the full-length GhWRKY15 CDNA}

Total RNA was extracted from the leaves of seven-dayold cotton seedlings $8 \mathrm{~h}$ after treatment with $2 \mathrm{mM} \mathrm{SA}$ treatment using Trizol Reagent (Invitrogen, USA). Reverse transcription-PCR (RT-PCR) and RACE-PCR were used to amplify the full-length GhWRKY15 cDNA. The general PCR procedures and primers (WQ1 and WQ2) are shown in Additional file 1 (Table S1 and Table S2), respectively. The PCR product was purified, cloned into the pMD18-T vector, and transformed into competent Escherichia coli cells for sequencing. The amino acid sequence alignment of GhWRKY15 and its homologues was conducted using BLAST (http://www.ncbi.nlm.gov/ blast) and DNAman software 5.2.2 (Lynnon Biosoft, Canada). A phylogenic analysis of the GhWRKY15 protein and other WRKYs was performed in MEGA using the neighbour-joining method.

\section{Amplification of the GhWRKY15 genomic sequence and promoter}

Genomic DNA was isolated from seedling leaves using the revised CTAB method. One pair of primers (WG1 and WG2), which was designed and synthesised based on the full-length GhWRKY15 cDNA, was used to amplify the GhWRKY15 genomic sequence using genomic DNA as the template. I-PCR was performed to obtain the promoter sequence. Three restriction endonucleases (DraI, TaqI and VspI) were used to digest the cotton seedling genomic DNA, and T4 DNA ligase (TaKaRa, China) was used to self-ligate the DNA fragments into circles, which were used as templates to amplify the promoter region. Three promoter fragments were amplified using six pairs of primers. Using nested PCR, the first fragment was obtained using two pairs of primers, Dra1/ Dra2 and Dra3/Dra4, and a DraI-digested template. Based on the obtained sequences, the primer pairs Taq1/ Taq2, Taq3/Taq4, Vsp1/Vsp2, and Vsp3/Vsp4 were used to sequentially amplify the other two fragments using the same method. The deduced portion of the promoter with the three fragments was subsequently verified using the special primers WP1 and WP2. The sequences of the primers are provided in Additional file 1 (Table S2). The programme PlantCARE (http://bioinformatics.psb. ugent.be/webtools/plantcare/html) was used to analyse the GhWRKY15 promoter sequence.

\section{Subcellular localisation of GhWRKY15}

To construct the pBI121-GhWRKY15-GFP expression plasmid driven by the cauliflower mosaic virus (CaMV) 
$35 \mathrm{~S}$ promoter, the coding sequence of GhWRKY15 without a termination codon was amplified using PCR and the primers Wsb-1 (5'-GGATCCCATCTTTTCCTAA TGTGGGAT-3', BamHI site underlined) and Wsb-2 (5'CTCGAGTGAAGATTCAAGTATGGTGG-3', XhoI site underlined). This fragment was subsequently fused to the N-terminus of GFP (green fluorescent protein). The resulting expression plasmid, pBI121-GhWRKY15-GFP, or the pBI121-GFP control plasmid was transformed into onion (Allium cepa) epidermis cells via biolistic bombardment transformation using the Biolistic PDS$1000 / \mathrm{He}$ system (Bio-Rad, USA) with gold particles $(1.0 \mu \mathrm{l})$ and a helium pressure of 1,350 psi. After incubation of the transformed onion epidermis cells at $25^{\circ} \mathrm{C}$ for $8 \mathrm{~h}$ in the dark, the nuclei were stained with $100 \mu \mathrm{g} /$ $\mathrm{ml}$ of 4',6-diamidino-2-phenylindole (DAPI) (Solarbio, China) in phosphate-buffered saline for $10 \mathrm{~min}$, and the onion epidermal cells were visualised using a fluorescence microscope (BX51, model 7.3; Japan).

\section{Northern blot analysis}

Total RNA was extracted using an RNeasy Mini Kit (Qiagen, USA), as recommended by the manufacturer, fractionated in a 1.0\% agarose gel containing formaldehyde and blotted onto a Hybond-N nylon membrane. A fragment amplified using the two specific primers WN1 and WN2, which were designed based on the GhWRKY15 cDNA sequence, was used as a probe, and northern blot hybridisations were performed as described by [37].

\section{Vector construction and transgenic plant generation}

The full-length coding sequence of GhWRKY15 amplified using the primers WE1 (5'-GGATCCCATCTTTTCC TAATGTGGGAT-3') with a BamHI site and WE2 (5'GAGCTCCTATGAAGATTCAAGTATGGTGG-3') with a SacI site was inserted into the same sites behind the CaMV35S promoter in the pBI121 binary vector. The ligated construct (pBI121-GhWRKY15) was electroporated into Agrobacterium tumefaciens (strain LBA4404) and transformed into tobacco (Nicotiana tabacum cv. NC89) using the leaf disk method as described previously [38]. The $\mathrm{T}_{0}$ and $\mathrm{T}_{1}$ transgenic tobacco plants were verified by PCR with special primers, which were designed according to the full-length coding sequence of GhWRKY15. The transgenic $\mathrm{T}_{2}$ lines were used in the experiments.

\section{Disease resistance of transgenic tobacco plants}

C. gossypii and P. parasitica spore suspensions $\left(10^{6}\right.$ conidia/ml) prepared in $1 \%$ glucose were sprayed onto the leaves of seven-week-old GhWRKY15 transgenic tobacco plants and wild-type plants. The inoculated plants were maintained in the growth chamber at $25^{\circ} \mathrm{C}$ for $24 \mathrm{~h}$ in the dark and were incubated at $25^{\circ} \mathrm{C}$ under a
$16 \mathrm{~h}$ light/8 h dark period. The inoculations with TMV and CMV were performed using a standard mechanical rubbing method, as described in [33]. The inoculated plants were cultured in the greenhouse at $25{ }^{\circ} \mathrm{C}$ under a $16 \mathrm{~h}$ light/8 h dark period.

\section{Virus quantitation}

Polyclonal antisera specific for the CP of TMV and CMV was used to detect virus accumulation in the tobacco leaves. The proteins in the inoculated leaves were extracted 10 days post-infection. Alkaline phosphataseconjugated goat anti-rabbit immunoglobulin G (Promega, USA) was used as the secondary antibody, and the plates were developed using a $p$-nitrophenyl phosphate (Sigma, USA) solution as a substrate. The absorbance of the developed plates was read at $490 \mathrm{~nm}$ using a Bio-Rad ELISA reader.

\section{Histochemical detection of $\mathrm{H}_{2} \mathrm{O}_{2}$ and enzyme activity assays}

Using 3,3-diaminobenzidine (DAB) as a substrate, $\mathrm{H}_{2} \mathrm{O}_{2}$ was visually detected in the leaves of the plants [39]. After various treatments, the leaves of the transgenic and wild-type tobacco plants were immersed in a $1 \mathrm{mg} /$ $\mathrm{ml}$ DAB solution for $6 \mathrm{~h}$ in the dark and subsequently treated with $95 \%$ ethanol to remove the chlorophyll. This treatment decolourises the leaves, except for the deep-brown stain produced by the polymerisation of DAB following interaction with $\mathrm{H}_{2} \mathrm{O}_{2}$. The leaves of the transgenic and WT plants inoculated with TMV or $C$. nicotianae were collected to measure the SOD, POD, CAT and APX activity, as described previously [40].

\section{Fluorescence real-time qPCR}

Fluorescence real-time qPCR was performed using cDNA as the template, which was synthesised from total RNA extracted from the transgenic or WT tobacco lines. The amplified fragments were all shorter than $300 \mathrm{bp}$. The qPCR technique was performed using the SYBR PrimeScript RT-PCR Kit (TaKaRa, China) and a CFX96TM RealTime System (Bio-Rad, USA), as recommended by the manufacturer. The PCR programme was as follows: predenaturation at $95^{\circ} \mathrm{C}$ for $30 \mathrm{~s} ; 40$ cycles of $95^{\circ} \mathrm{C}$ for $30 \mathrm{~s}, 53^{\circ}$ $\mathrm{C}$ for $15 \mathrm{~s}$ and $72^{\circ} \mathrm{C}$ for $15 \mathrm{~s}$; and a melt cycle from $65^{\circ} \mathrm{C}$ to $95^{\circ} \mathrm{C}$. The expression of actin was used as an internal control. The significance of the differences was determined using Statistical Analysis System (SAS) version 9.1 (SAS Institute, USA). To determine the copy number, qPCR was also used, as described by [41].

\section{Expression analysis of defence-related genes in transgenic and WT lines}

The qPCR technique was used to analyse the expression of PR genes, ET biosynthesis-related genes and oxidation- 
related genes. The GenBank accession numbers of the defence-related genes examined in the qPCR analysis are as follows: X63603 (actin), X06361 (NtPR1), M60460 (NtPR2), X58546 (NtPR4), M29279 (NtPR5), DQ837218 (NtNPR1), NTU96152 (SAR8.2 $\left.2^{\circ}\right), \quad$ AB012857 (NtACO), AJ005002 (NtACS) AB093097, (NtSOD), AU15933 (NtAPX1), D85912 (NtAPX2), U93244 (NtCAT1), AB041518 (NtGPX) and AF454759 (NtCA).

\section{Additional files}

Additional file 1: Table S1. Polymerase chain reaction amplification conditions. Table S2 Primers used in this study. Table S3 Ct and Tm value of GhRDR6 and GhWRKY15 genes in cotton. Table S4 Estimation of copy number of GhWRKY15 gene in cotton.

Additional file 2: Figure S1. Schematic representation of the locus. The lengths of the exons and introns of GhWRKY15 (GenBank accession number: GU207869), AtWRKY7 (NC_003075), AtWRKY15 (NC_003071) and VVWRKY (NW_002239918) are indicated according to the scale below. Exons and introns are designated using white or gray bars, respectively. The untranslated regions are indicated by black bars. The translation initiation and stop codons are marked with $(\triangle)$ and $(\boldsymbol{\nabla})$, respectively. The R-type introns are indicated by $\left(^{*}\right)$.

Additional file 3: Figure S2. Standard curves of GhRDR6 and GhWRKY15. (A) Standard curves of GhRDR6 gene from the amplification of six five-fold serial dilutions of plasmid fused by GhRDR6. (B) Standard curves of GhWRKY15 gene from the amplification of six five-fold serial dilutions of the same plasmid fused by GhWRKY15. Correlation coefficient and slope values are indicated. The calculated threshold cycle values were plotted versus the log of each starting quantity.

Additional file 4: Figure S3. Relative expression of GhWRKY15 in response to different fungal infections and hormone treatments. The results correspond to the results in Figure 3 . Transcriptional levels of GhWRKY15 under different fungal infections and hormone treatments are indicated relative to the level of wild-type cottons without any treatment taken as 1 in each experiment.

Additional file 5: Figure S4. MV enhances GhWRKY15 expression. The result corresponds to the results in Figure $7 D$. Transcriptional levels of GhWRKY15 under $0.5 \mathrm{mM} \mathrm{MV}$ treatment are indicated relative to the level of wild-type cottons without any treatment taken as 1 .

\section{Authors' contributions}

FY carried out most of the experiments, and drafted the manuscript. YH participated in RNA blot analysis and function analysis of the transgenic plants. WL helped to revise the manuscript. CW, XC and XG conceived the experimental design and helped to draft the manuscript. All authors read and approved the final manuscript.

\section{Acknowledgments}

This work was financially supported by the National Natural Science Foundation of China (No. 31171837) and Genetically Modified Organisms Breeding Major Projects of China (2009ZX08009-092B).

\section{Received: 2 February 2012 Accepted: 4 June 2012}

Published: 12 August 2012

\section{References}

1. Skriver K, Mundy J: Gene expression in response to abscisic acid and osmotic stress. Plant Cell 1990, 2:503-512.

2. Rushton PJ, Somssich IE: Transcriptional control of plant genes responsive to pathogens. Curr Opin Plant Biol 1998, 1:311-315.

3. Yu D, Chen C, Chen Z: Evidence for an important role of WRKY DNA binding proteins in the regulation of NPR1 gene expression. Plant Cell 2001, 13:1527-1540.

4. Eulgem T, Rushton PJ, Robatzek S, Somssich IE: The WRKY superfamily of plant transcription factors. Trends Plant Sci 2000, 5:199-206.
5. Ross CA, Liu Y, Shen QJ: The WRKY gene family in rice (Oryza sativa). J Integr Plant Biol 2007, 49:827-842.

6. Rushton PJ, Somssich IE, Ringler P, Shen QJ: WRKY transcription factors Trends Plant Sci 2010, 15:248-258.

7. Li J, Brader G, Palva ET: TheWRKY70 transcription factor: a node of convergence for jasmonate-mediated and salicylate-mediated signals in plant defense. Plant Cell 2004, 16:319-331.

8. Oh SK, Baek KH, Park JM, Yi SY, Yu SH, Kamoun S, Choi D: Capsicum annuum WRKY protein CaWRKY1 is a negative regulator of pathogen defense. New Phytol 2008, 177:977-989.

9. Murray SL, Ingle RA, Petersen LN, Denby KJ: Basal resistance against Pseudomonas syringae in Arabidopsis involves WRKY53 and a protein with homology to a nematode resistance protein. Mol Plant Microbe Interact 2007, 20:1431-1438.

10. Higashi $K$, Ishiga $Y$, Inagaki $Y$, Toyoda $K$, Shiraishi $T$, Ichinose $Y$ : Modulation of defense signal transduction by flagellin-induced WRKY41 transcription factor in Arabidopsis thaliana. Mol Genet Genomics 2008, 279:303-312.

11. Yang P, Chen C, Wang Z, Fan B, Chen Z: A pathogen- and salicylic acidinduced WRKY DNA-binding activity recognizes the elicitor response element of the tobacco class I chitinase gene promoter. Plant J 1999, 18:141-149.

12. Boland JF, Linthorst HJM: Plant pathogenesis-related protein induced by virus infection. Annu Rev Phytopathol 1990, 28:113-138.

13. Zhou QY, Tian AG, Zou HF, Xie ZM, Lei G, Huang J, Wang CM, Wang HW, Zhang JS, Chen SY: Soybean WRKY-type transcription factor genes, GmWRKY13, GmWRKY21, and GmWRKY54, confer differential tolerance to abiotic stress in transgenic Arabidopsis plants. Plant Biotechnol J 2008, 6:486-503.

14. Xu X, Chen C, Fan B, Chen Z: Physical and functional interactions between pathogen-induced Arabidopsis WRKY18, WRKY40, and WRKY60 transcription factors. Plant Cell 2006, 18:1310-1326.

15. Xie Z, Zhang ZL, Zou X, Huang J, Ruas P, Thompson D, Shen QJ: Annotations and functional analyses of the rice WRKY gene superfamily reveal positive and negative regulators of abscisic acid signaling in aleurone cells. Plant Physiol 2005, 137:176-189.

16. Xie Z, Zhang ZL, Zou X, Yang G, Komatsu S, Shen QJ: Interactions of two abscisic-acid induced WRKY genes in repressing gibberellin signaling in aleurone cells. Plant J 2006, 46:231-242.

17. Lagace $M$, Matton DP: Characterization of a WRKY transcription factor expressed in late torpedo-stage embryos of Solanum chacoense. Planto 2004, 219:185-189.

18. Miao $Y$, Laun T, Zimmermann P, Zentgraf U: Targets of theWRKY53 transcription factor and its role during leaf senescence in Arabidopsis. Plant Mol Biol 2004, 55:853867.

19. Wang H, Avci U, Nakashima J, Hahn MG, Chen F, Dixon RA: Mutation of WRKY transcription factors initiates pith secondary wall formation and increases stem biomass in dicotyledonous plants. Proc Natl Acad Sci U S A 2010, 107:22338-22343.

20. Park CY, Lee JH, Yoo JH, Moon BC, Choi MS, Kang YH, Lee SM, Kim HS, Kang KY, Chung WS, Lim CO, Cho MJ: WRKY group IId transcription factors interact with calmodulin. FEBS Lett 2005, 579:1545-1550.

21. Wang M, Li S, Yang H, Gao Z, Wu C, Guo X: Characterization and functional analysis of GhRDR6, a novel RDR6 gene from cotton (Gossypium hirsutum L.). Biosci Rep 2012, 32:139-151.

22. Menke FL, Kang HG, Chen Z, Park JM, Kumar D, Klessig DF: Tobacco transcription factor WRKY1 is phosphorylated by the MAP kinase SIPK and mediates HR-like cell death in tobacco. Mol Plant Microbe Interact 2005, 18:1027-1034.

23. Kende H: Ethylene biosynthesis. Annu Rev Plant Physiol Plant Mol Biol 1993, 44:283-307

24. Hernández JA, Rubio M, Olmos E, Ros-Barceló A, Martínez-Gómez \P. Oxidative stress induced by long-term PlumPox virus infection in peach (Prunus persica). Physiol Plant 2004, 122:486-495.

25. Vyas D, Kumar S: Purification and partial characterization of a low temperature responsive $\mathrm{Mn}-\mathrm{SOD}$ fromtea (Camellia sinensis (L) O Kuntze). Biochem Biophys Res Commun 2005, 329:831-838.

26. Noctor $\mathrm{G}$, Foyer $\mathrm{CH}$ : Ascorbate and glutathione: keeping active oxygen under control. Annu Rev Plant Physiol Plant Mol Biol 1998, 49:249-279. 
27. Guo R, Yu F, Gao Z, An H, Cao X, Guo X: GhWRKY3, a novel cotton (Gossypium hirsutum L) WRKY gene, is involved in diverse stress responses. Mol Biol Rep 2010, 38:49-58.

28. Tao Z, Liu H, Qiu D, Zhou Y, Li X, Xu C, Wang S: A pair of allelic WRKY genes play opposite roles in rice-bacteria interactions. Plant Physiol 2009, 151:936-948.

29. Turck F, Zhou A, Somssich IE: Stimulus-dependent, promoter-specific binding of transcription factor WRKY1 to its native promoter and the defense-related gene PcPR1-1 in Parsley. Plant Cell 2004, 16:2573-2585.

30. Dong J, Chen C, Chen Z: Expression profiles of the Arabidopsis WRKY gene superfamily during plant defense response. Plant Mol Biol 2003, 51:21-37.

31. Kotchoni SO, Gachomo EW: The reactive oxygen species network pathways: an essential prerequisite for perception of pathogen attack and the acquired disease resistance in plants. J Biosci 2006, 31:389-404.

32. Zhang L, Xi D, Luo L, Meng F, Li Y, Wu CA, Guo X: Cotton GhMPK2 is involved in multiple signaling pathways and mediates defense responses to pathogen infection and oxidative stress. FEBS J 2011, 278:1367-1378.

33. Shi J, An HL, Zhang L, Gao Z, Guo XQ: GhMPK7, a novel multiple stressresponsive cotton group C MAPK gene, has a role in broad spectrum disease resistance and plant development. Plant Mol Biol 2010, 74:1-17.

34. Wu L, Zhang Z, Zhang H, Wang XC, Huang R: Transcriptional modulation of ethylene response factor protein JERF3 in the oxidative stress response enhances tolerance of tobacco seedlings to salt, drought, and freezing. Plant Physiol 2008, 148:1953-1963.

35. Shekhawat UK, Ganapathi TR, Srinivas L: Cloning and characterization of a novel stress-responsive WRKY transcription factor gene (MusaWRKY71) from Musa spp. cv. Karibale Monthan (ABB group) using transformed banana cells. Mol Biol Rep 2011, 38:4023-4035.

36. Zhang ZL, Xie Z, Zou X, Casaretto J, Ho TH, Shen QJ: A rice WRKY gene encodes a transcriptional repressor of the gibberellin signaling pathway in aleurone cells. Plant Physiol 2004, 134:1500-1513.

37. Guo YH, Yu YP, Wang D, Wu CA, Yang GD, Huang JG, Zheng CC: GhZFP1, a novel CCCH-type zinc finger protein from cotton, enhances salt stress tolerance and fungal disease resistance in transgenic tobacco by interacting with GZIRD21A and GZIPR5. New Phytol 2009, 183:62-75.

38. Horsch RB, Fry JE, HoVmann NL, Eichholtz D, Rogers SG, Fraley RT: A simple and general method for transferring genes into plants. Science 1985, 227:1229-1231.

39. Orozco-Cardenas M, Ryan CA: Hydrogen peroxide is generated systemically in plant leaves by wounding and systemin via the octadecanoid pathway. Proc Natl Acad Sci U S A 1999, 96:6553-6557.

40. Li Q, Yu B, Gao Y, Dai A, Bai J: Cinnamic acid pretreatment mitigates chilling stress of cucumber leaves through altering antioxidant enzyme activity. J plant physiol 2010, 168:927-934.

41. Mason G, Provero P, Vaira AM, Accotto GP: Estimating the number of integrations in transformed plants by quantitative real-time PCR. BMC Biotechnol 2002, 2:20.

doi:10.1186/1471-2229-12-144

Cite this article as: Yu et al:: GhWRKY15, a member of the WRKY transcription factor family identified from cotton (Gossypium hirsutum L.), is involved in disease resistance and plant development. BMC Plant Biology 2012 12:144.

\section{Submit your next manuscript to BioMed Central and take full advantage of:}

- Convenient online submission

- Thorough peer review

- No space constraints or color figure charges

- Immediate publication on acceptance

- Inclusion in PubMed, CAS, Scopus and Google Scholar

- Research which is freely available for redistribution 